Ann. Biol. anim. Bioch. Biophys., I974, 14 (2), 229-237.

\title{
LES PROTÉASES DIGESTIVES CHEZ UN TÉLÉOSTÉEN CARNIVORE DICENTRARCHUS LABRAX
}

\author{
Élisabeth ALLIOT, A. FEBVRE et R. MÉTAILLER \\ Station marine d'Endoume, \\ 13007 Marseille
}

RÁSUMÉ

L'activité protéolytique a été mesurée dans des extraits de tractus digestif de Dicentrarchus labrax. Elle présente des optima aux environs de $\mathrm{pH} 9$ et $\mathrm{pH}$ 2, avec l'hémoglobine comme substrat. Le $\mathrm{pH}$ du suc digestif varie entre $\mathrm{pH} 2$ et 6 dans l'estomac, et entre 7 et 9 dans l'intestin.

La forte activité dans la zone acide est trouvée essentiellement avec les extraits d'estomac : l'enzyme présente peut être considérée comme analogue à la pepsine.

Dans la zone alcaline, les enzymes actives hydrolysent les substrats de la trypsine, de la chymotrypsine, des carboxypeptidases A et B, et de l'élastase. Les activités sont plus élevées pour les extraits de cœca pyloriques que d'intestin. L'activité vis-à-vis des substrats de la trypsine est inhibée par l'inhibiteur de la trypsine du soja, et celle des carboxypeptidases par l'EDTA.

Dans le cadre d'une étude de la nutrition du loup ou bar, Dicentrarchus labrax, il paraissait nécessaire d'avoir une estimation de la nature et de la répartition des enzymes élaborées dans le tractus digestif de ces animaux. De nombreux travaux effectués chez les poissons (CREAC'H, I963), montrent qu'il existe des variations dues à l'espèce, au régime alimentaire et aux divers paramètres physico-chimiques. Le loup étant un poisson carnivore, ce qui suppose un apport protéique important dans son alimentation, une revue des principales enzymes protéolytiques a été entreprise pour servir de support à des recherches ultérieures sur la digestibilité des diverses protéines susceptibles d'être ingétées par l'animal.

\section{MATÉRIEL, E'T MÉTHODES}

Les spécimens de Dicentrarchus labrax ont été pêchés à la senne ou à l'épervier dans la région marseillaise, de mai à septembre I97I, et ramenés vivants au laboratoire. 


\section{Préparation des extraits}

Des individus de longueur totale comprise entre Io et 40 centimètres ont servi pour cette étude. Certains sont sacrifiés dès leur arrivée au laboratoire; d'autres sont conservés en circuit ouvert et nourris avec du poisson haché, des moules ou des crevettes. Les animaux sont légèrement anesthésiés au MS 222 (Sandoz), et le tube digestif est conservé à $0^{\circ} \mathrm{C}$ immédiatement après le prélèvement. Les contenus stomacaux sont rapidement examinés afin de déterminer l'état nutritionnel du poisson. Sur les plus gros individus, lorsque cela est possible, du suc digestif est prélevé à l'aide d'une seringue pour une lecture de $\mathrm{pH}$ (Microélectrode Metrohm). Le plus souvent le $\mathrm{pH}$ est déterminé avec du papier indicateur (Merck).

Le tube digestif présente trois parties bien distinctes : un estomac, des cœea pyloriques et un intestin court. Le pancréas est diffus et localisé dans le mésentère autour des coeca et de l'intestin antérieur. Les différentes portions ainsi prises en considération sont rincées, pesées et broyées dans de l'eau distillée, avec un broyeur de type Potter-Elvejhem, ou de type UltraTurrax dans le cas de l'estomac. Sur les plus gros individus, les muqueuses gastriques et intestinales, raclées au scalpel, ont servi à la préparation des extraits. L'extraction est réalisés à $4^{\circ} \mathrm{C}$, pendant 24 heures, sous agitation magnétique. Les extraits sont centrifugés à 6000 r.p.m. pendant Io minutes.

\section{Dosage des activités enzymatiques}

Pour l'activité protéolytique globale: méthode d'ANson (I938) avec l'hémoglobine comme substrat, méthode de KUNITz (1947) avec la caséine comme substrat.

Pour la pepsine : méthode d'Anson telle qu'elle est décrite par HerRiott (1955), avec l'hémoglobine ( $2 \mathrm{p}$. Ioo) ajustée à $\mathrm{pH}$ 2. L'activité globale de la pepsine et du pepsinogène est déterminée.

Pour la trypsine et la chymotrypsine : méthode de Hummex. (I959), en employant des substrats (TAME-BTEE) spécifiques. Le dosage est réalisé par titration automatique.

Pour la carboxypeptidase A : méthode de LAcko et Neurath (I970) avec l'acide HippurylL-phénylallactique comme substrat. L'activité est mesurée par titration automatique.

Pour la carboxypeptidase B : méthode de Folk et al. (1960).

Pour l'élastase : méthode de MANDL (I962).

\section{Dosage des protéines}

La méthode de Lowny et al. (195I) a été utilisée pour le dosage des protéines dans les extraits.

\section{RÉSULTATS}

\section{Influence des conditions de pH sur l'activité protéolytique}

Les $\mathrm{pH}$ estimés dans les différentes portions du tube digestif chez les individus conservés au laboratoire, montrent des variations consécutives au repas. Dans les premières heures qui suivent l'ingestion, le $\mathrm{pH}$ du suc gastrique s'abaisse aux environs de 2. Dans la partie duodénale, il est proche de 7 ; il devient de plus en plus alcalin vers la zone postérieure du tube digestif où il est voisin de 7,6-8. $48 \mathrm{~h}$ après un repas, dans l'estomac, le pH est compris entre 6 et 7 ; au niveau des coca pyloriques, ainsi que dans le fluide intestinal, il est voisin de 8 (tab1. r).

Les conditions optimales de $\mathrm{pH}$ pour l'activité protéolytique globale ont été étudiées pour les différentes parties du tractus digestif, avec l'hémoglobine comme substrat. Les résultats montrent qu'il existe au moins deux groupes de protéases différentes : les unes avec un optimum vers $\mathrm{pH} 2$, les autres avec un optimum compris entre 8 et ro (fig. I). Les enzymes actives dans la zone acide, vraisemblablement de même nature que la pepsine, sont trouvées dans les extraits d'estomac. 


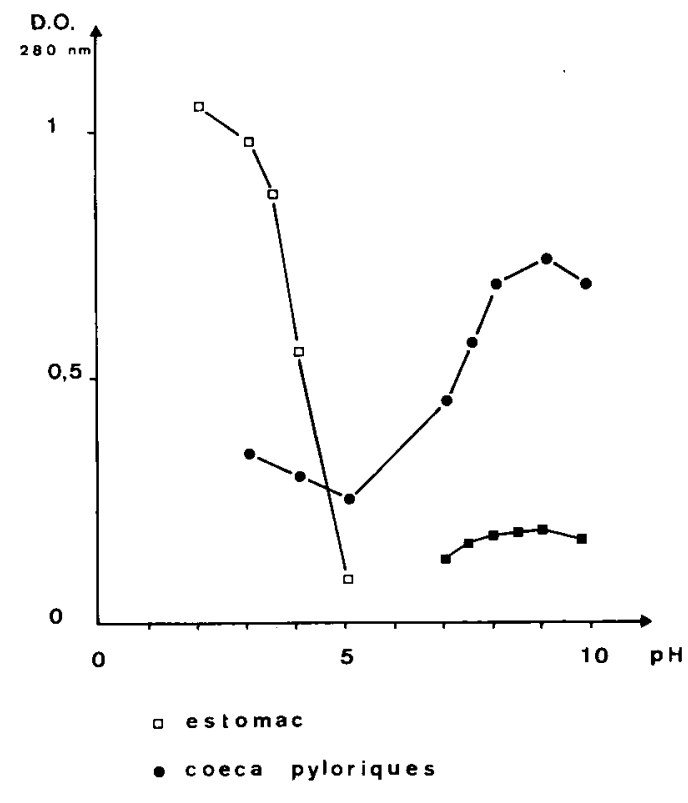

- intestin

FIG. I. - Influence du $p H$ sur l'activité protéolytique globale, a $25^{\circ} \mathrm{C}$ Substrat : Hémoglobine

\section{TABLEAU I}

Évolution $d u p H$ dans le fluide intestinal après un repas

\begin{tabular}{|c|c|c|c|c|c|}
\hline \multirow{2}{*}{ Organe } & \multicolumn{5}{|c|}{ Temps écoulé après l'ingestion d'un repas } \\
\hline & $3 \mathrm{~h}$ & $6 \mathrm{~h}$ & $12 \mathrm{~h}$ & $24 \mathrm{~h}$ & $48 \mathrm{~h}$ \\
\hline Estomac .... & $2,1-2,3$ & $2,5-2,7$ & $5,5-5,8$ & $6,1-6,4$ & $6,{ }_{4}^{4}-6,7$ \\
\hline $\begin{array}{l}\text { Intestin : } \\
\text { antérieur .. }\end{array}$ & $6,7-7,0$ & $7,3-7,6$ & $7,4-7,6$ & $7,6-7,9$ & $7,9-8,0$ \\
\hline postérieur . . & 7,6 & $7,8-7,9$ & $7,9-8,1$ & $8,3-8,5$ & $8,3-8,5$ \\
\hline
\end{tabular}


La protéolyse dans la zone alcaline est observée pour les extraits de coeca pyloriques et d'intestin (à un degré bien moindre pour ces derniers). Les extraits de cœeca pyloriques ont été étudiés dans les mêmes conditions avec la caséine comme substrat.

\section{Influence des conditions de température sur l'activité protéolytique globale}

Les conditions de température ont été étudiées entre $10^{\circ}$ et $40^{\circ} \mathrm{C}$. Avec un substrat caséine comme avec l'hémoglobine, on obtient un large pic d'activité entre $30^{\circ}$ et $40^{\circ} \mathrm{C}$. Les expériences ultérieures ont été menées à $25^{\circ} \mathrm{C}$, température qui se trouve dans la partie linéaire de la courbe et proche de celle du milieu à l'époque des pêches (Êtangs de Camargue).

\section{Activité dans les différentes parties du tractus digestif}

Les tests ultérieurs ont été effectués en vue de déterminer la nature et l'activité des enzymes dans les zones de $\mathrm{pH}$ optimum.

\section{L'activité protéolytique aux environs de $p H 2$.}

Une activité protéolytique importante peut être mesurée avec le suc gastrique et les extraits de muqueuse stomacale. Les extraits de muqueuse ajustés à $\mathrm{pH} z$ préalablement aux essais montrent une activité accrue. L'activité des extraits de muqueuse stomacale a été comparée à celle obtenue avec de la pepsine purifiée (Pepsine I : Io ooo Sigma) : chez les individus adultes, l'activité mesurée correspond à celle obtenue avec $70-75 \mathrm{mg}$ de pepsine. Chez les plus petits individus, où l'estomac entier a été homogénéisé, les variations individuelles sont très importantes : un gramme de poids frais donne des activités comparables à celle de $42 \mathrm{mg}$ de pepsine $(42 \pm \mathrm{I} 9$, $n=7)$.

\section{L'activité protéolytique dans la zone alcaline.}

L'analyse de l'activité globale a montré que la plupart des enzymes se trouvent s ous forme active dans les extraits. Ceci n'exclut pas le fait que les enzymes puissent ềre élaborées sous forme de zymogènes, mais le processus d'extraction rend sans doute impossible leur isolement (ZENDZIAN et BERNARD, I967).

\section{Trypsine.}

L'activité vis-à-vis du TAME, substrat spécifique de la trypsine, des extraits de cœea pyloriques, d'intestin et de fluide intestinal, a été étudiée. Le maximum d'hydrolyse du TAME est obtenu avec les extraits de cœca ; la muqueuse ne présente en comparaison qu'une activité environ dix fois plus faible. Dans tous les cas, la courbe d'activité en fonction du $\mathrm{pH}$ présente un maximum compris entre $\mathrm{pH} 8,2$ et 9,0 (fig. 2). Les enzymes semblent relativement stables en milieu alcalin : on n'enregistre aucune perte d'activité lorsque les extraits sont conservés à $+4^{\circ} \mathrm{C}$ à pH 8,5 pendant 24 heures.

L'hydrolyse du TAME est ralentie et presque totalement supprimée lorsqu'on ajoute des quantités croissantes d'inhibiteur de la trypsine du soja dans le milieu 
réactionnel ; cela tend à montrer que cette enzyme est de nature analogue à la trypsine des mammifères et que les poissons sont sensibles à cet inhibiteur.

L'activité estérolytique des extraits a été comparée à l'activité de quantités connues de trypsine de pancréas de bœuf (Kochlight and Co 5222 t). Les extraits issus d'un gramme, en poids frais, de cœea pyloriques ont une activité équivalente à 4,68 $\pm \mathrm{I}, 97 \mathrm{mg}$ de trypsine purifiée, à $\mathrm{pH} 8$,o. Les extraits d'intestin présentent des activités équivalentes à 300-600 $\mu \mathrm{g}$ de trypsine par gramme de poids frais.

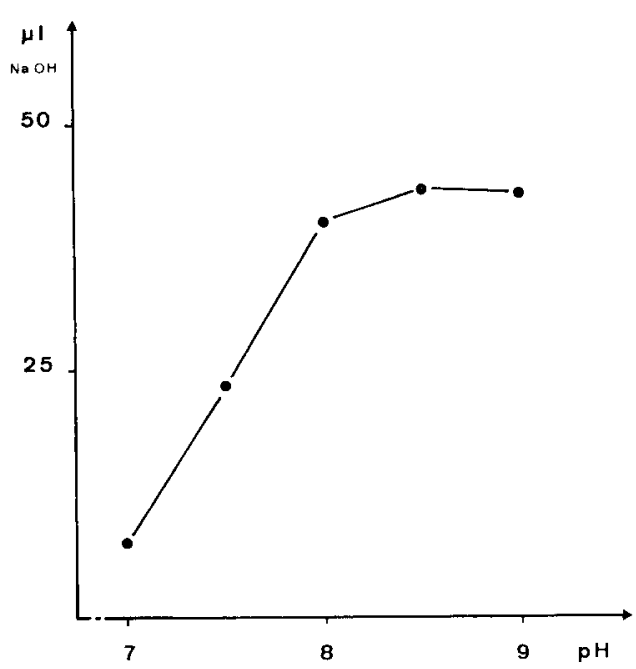

FIG. 2. - Influence du pH sur l'activité trypsique des extraits de coeca pyloriques

Substrat : TAME

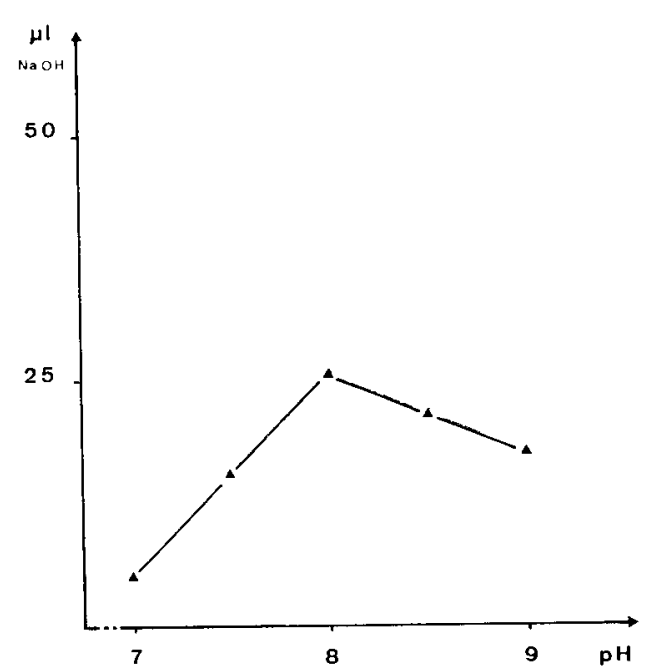

$\mathrm{Fig}_{\mathrm{r} .3}$ 3. - Influence du pH sur l'activité chymotrypsique des extraits de coeca pyloriques

Substrat : BTEE

\section{Chymotrypsine.}

L'activité des extraits vis-à-vis du BTEE, substrat de la chymotrypsine, a été testée. Comme pour la trypsine, 1'activité maximale est observée pour les extraits de cœea pyloriques. L'optimum de $\mathrm{pH}$ pour 1'hydrolyse du BTEE se situe entre 8,0 et 8,2 (fig. 3 ).

L'activité des extraits a été comparée à celle obtenue avec de la chymotrypsine de pancréas de bœuf (Calbiochem). A pH 8,0, l'activité d'un gramme de poids frais de coeca pyloriques équivaut à celle obtenue avec $3, \mathbf{I} 2 \pm \mathrm{I}, 56 \mathrm{mg}$ de chymotrypsine. Pour l'intestin l'activité est beaucoup plus faible : $160-380 \mu \mathrm{g}$.

\section{Carboxypeptidases.}

L'hydrolyse de 1'acide hippuryl-L-phénylallactique est réalisée par les extraits de cœca pyloriques. L'activité à $\mathrm{pH} 8,5$ est plus élevée qu'à $\mathrm{pH} 8$,o. Ellle a été comparée à celle obtenue avec de la carboxypeptidase de bœuf purifiée (Calbiochem). L'activité mesurée par gramme de poids frais équivaut à celle obtenue avec $\mathrm{I}, 24 \pm 0,72 \mathrm{mg}$ de carboxypeptidase à $\mathrm{pH} 8,0$. Lorsque les extraits sont incubés une heure à $20^{\circ} \mathrm{C}$ avec de l'EDTA ( IO $^{-2} \mathrm{M}$ ), 80 à $90 \mathrm{p}$. Ioo de l'activité est inhibée, laissant penser qu'il s'agit bien d'une métallo-enzyme. 
I a présence d'une enzyme analogue à la carboxypeptidase $B$ a été recherchée : les extraits de cœca hydrolysent l'hippuryl-L-arginine, substrat classique pour cette enzyme.

\section{Élastase.}

Les extraits de cœca hydrolysent l'Elastine-orcéine. L'activité n'a pu être mesurée en l'absence d'étalon élastase. L'activité est plus élevée à $\mathrm{pH} 9,3$ qu'à $\mathrm{pH}$ 8,4 ; par contre, la cinétique d'activité (fig. 4) semble montrer une meilleure stabilité à- $\mathrm{pH} 8,4$.

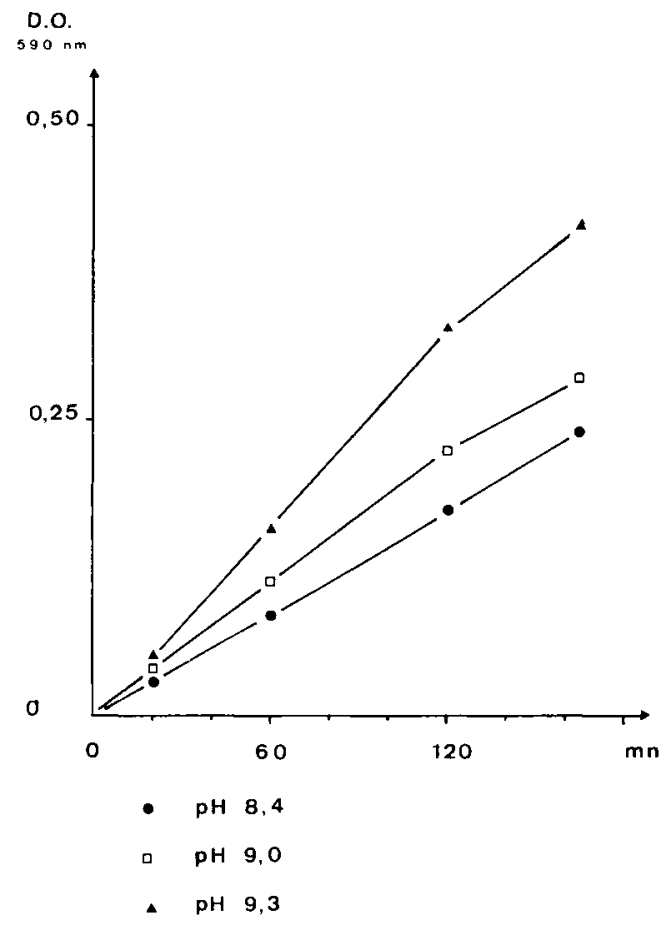

FIc. 4. -- Infuence du pH sur l'activité de l'élastase des exiraits de coca pyloriques Substrat : Élastine-Orcćine

\section{DISCUSSION E'T CONCLUSION}

Ces résultats montrent qu'il existe dans le tractus digestif du loup une activité protéolytique due à des enzymes analogues à celles des mammifères. Ceci concorde avec les résultats obtenus chez d'autres téléostéens (Revue par BERNARD, I952; VONK, I955; CREAC'H, I963). L'activité protéolytique dans l'estomac serait principalement due à une enzyme de même nature que la pepsine. NorRis et ÉLAM (I940), et Norris et Mathres (I953) ont montré, après isolement de la pepsine de saumon et de thon, que l'activité spécifique était très élevée. Il est possible que d'autres 
enzymes, telles que des cathepsines, soient également présentes dans l'estomac, comme Buchs (I954) l'a montré chez d'autres poissons.

L'activité protéolytique dans la zone alcaline semble être due principalement à des enzymes de même nature que la trypsine et la chymotrypsine. Croston (I960) a pu mettre en évidence ces deux types d'activité dans les cœca pyloriques de saumon, avec des pH optimaux aux environs de 9,0 ; il a également montré l'inhibition de l'activité de la trypsine par le facteur antitrypsique du soja. NiLsson et FANGE (I969I970), chez la Chimère et la Myxine, OosHrro (I97I) chez le Maquereau, et ZENDZIAN et BERNARD (I967) chez plusieurs espèces de poissons ont trouvé des résultats analogues. Cependant, comme le soulignent ZENDZIAN et BERNARD, 1'activité protéolytique est également due à la présence de carboxypeptidases et d'élastase. L'activité des dipeptidases, aminopeptidases, non citées dans cette étude, est aussi à prendre en considération.

Il est possible que l'activité observée dans la zone acide, avec les extraits de cœeca pyloriques, soit due à une contamination par des enzymes gastriques.

Les variations de $\mathrm{pH}$ in situ observées concordent avec les données de la littérature (BERNARD, I952). On peut penser que dans l'estomac existent les conditions optimales d'activité de la pepsine au moment de la digestion. Toutefois, la majorité des déterminations a été faite au contact de la muqueuse, et il est vraisemblable que l'acidité soit moins marquée à l'intérieur du bol alimentaire, comme cela a été montré chez le Brochet (VoNk, I955).

L'activité élevée, enregistrée au niveau des cœca pyloriques, est sans doute due au tissu pancréatique homogénéisé avec les cœca ; le pancréas serait responsable en grande partie de l'élaboration des enzymes actives dans la zone alcaline. Celles-ci gagnent le fluide intestinal, où elles trouvent des conditions de $\mathrm{pH}$ favorables. Il ne semble pas que la muqueuse intestinale joue un grand rôle dans la sécrétion des enzymes étudiées, d'autant plus que les procédés d'extraction (rinçage rapide de la muqueuse à l'eau distillée) obligent à prendre en considération les enzymes d'origine bactérienne. BIANCHI (I972, comm. pers.) a pu mettre en évidence des bactéries qui possèdent une activité caséinolytique. D'autre part, une étude histologique (VEGASVELEZ, I972) n'a pas permis de déceler des glandes sécrétrices dans la muqueuse intestinale chez cette espèce. Cette situation semble se retrouver chez de nombreux poissons.

Aucune purification d'enzymes n'ayant été réalisée au cours de cette étude, les activités exprimées n'ont qu'une valeur tout à fait relative, et sont données à titre d'indication. De plus, les activités notées, tant pour les extraits d'estomac que pour les extraits de cœeca, présentent des fluctuations importantes ; les paramètres faisant varier l'activité enzymatique sont nombreux et difficiles à contrôler chez les poissons. Différents auteurs ont montrél'influence du jê̂ne sur la sécrétion d'enzymes digestives ; Kitamikado et TaChivo (I960) ont trouvé des différences notables entre des poissons de tailles différentes; Aranicsev (I959), Chépik (I966), Kawashida (I952), ont pu mettre en évidence des variations saisonnières; par ailleurs, NaGAYama et SAITo (I969), NAGASE (I964) ont montré qu'il existait un rapport entre cette sécrétion et la teneur en protéines de l'alimentation. Des études sont en cours pour déterminer l'influence de certains de ces paramètres sur l'activité sécrétrice chez le Loup. 


\section{REMERCIEMENTS}

Ce travail a été effectué à la Station Marine d'Endoume à Marseille, grâce à un contrat entre le C.N.E. X. O. et l'U. E. R, des Sciences de la Mer et de l'Environnement de MarseilleLuminy.

\section{SUMMARY}

\section{DIGESTIVE PROTEASES IN A CARNIVOROUS TELEOST, DICENTRARCHUS LABRAX}

Proteolytic activity was observed in extracts of the digestive tract of Dicentrarchus labrax. It shows optima of about $\mathrm{pH} 2$ and $\mathrm{pH} 9$ with hemoglobin as substrate (fig. $\mathbf{r}$ ). The $\mathrm{pH}$ of digestive fluid fluctuates between $\mathrm{pH} 2$ and 6 in the stomach, and between 7 and 9 in the intestine (table r).

Stomach extracts mainly show strong activity in the acidic range. The enzyme may be considered as analogous to pepsin.

In the alcaline range, trypsin, chymotrypsin, carboxypeptidases and elastase-like activities are observed. The activities recorded are higher in extracts from the pyloric coca and pancreas area than from the intestine.

Trypsin-like activity shows an optimum of about $\mathrm{pH} 8.5$ (fig. 2) and is inhibited by soybean trypsin inhibitor.

Optimum $\mathrm{pH}$ for chymotrypsin-like activity is about $\mathrm{pH} 8.2$ (fig. 3). Elastase and carboxypeptidase-like activities are higher with more alcaline $\mathrm{pH}$ (fig. 4). Carboxypeptidase activity is inhibitad by EDTA.

\section{RÉFÉRENCES BIBLIOGRAPHIQUES}

Ananicsev A. V., r959. Digestive enzymes of fish and seasonal change of their activity. Biochimija., 24, I033-1040.

Anson M. L., I938. The estimation of pepsin, trypsin, papain and cathepsin whith hemoglobin. J.gen. Physiol., 22, 79-89.

Bernard F., 1952. La digestion chez les poissons. Trav. Lab. Hydrob. Pisciculture, Univ., Grenoble, 44, 6I-95.

Buchs S., I954. Der proteolyse in magen. Z. Vergl. Physiol., 36, I65-175.

Cнерік L., I 966 . Activity of Carp digestive enzymes at different seasons of the year. Biol. Abstr., 47,60747 .

Creac'h P. V., r963. Les enzymes protéolytiques des poissons. Ann. Nutr. Alim., 17, 375-47I.

Croston C. B., 1960. Tryptic enzymes of Chinook Salmon. Arch. Biochem. Biophys., 89, 202-2o6.

Folk J. E,, Piez K. A., Carrol W. R., Gladner J. A., I96o. Carboxypeptidase B. J. Biol. Chem., 235, I393-r399.

Herriots R. M., r955. Swine Pepsin and Pepsinogen. In Methods in Enzymology. Colowick et Kaplan Éd. II, 3-7, Acad. Press, New York.

Hummer B.C. W., r959. A modified spectrophotometric determination of chymotrypsin, trypsin and thrombin. Canad. J. Biochem. Physiol., 37, I393-1400.

KaWASHIDA K. S., I952. Studies on the enzymes of Skipjack : Katsuronus vagans entrails. I. On the seasonal variation of proteolytic enzymes activity in pyloric coeca. Bull. Jap. Soc. sci. Fish., 18, r51I54.

Kitamikado M., Tachino S., I960. Studies on the digestive enzymes of rainbow trout. II. Proteases. Buil. Jap. Soc. sci. Fish., 26 (7), 685-69o.

Kunitz M., I947. Cristalline soybean trypsin inhibitor. II. General properties. J. Biol. Chem., 30, 29I-3IO.

Lacko A. G., Neurath M., I970. Studies on procarboxypeptidase A of the spiny dogfish (Squalus acanthias). Biochemistry, 9, I680-1690.

Lowry O. H., Rosebough N. J., Fark A., Randall R. J., I95x. Protein measurement with the Folin-phenol reagent. J. Biol. Chem., 193, 265-275. 
Mandl I., 1962. Pancreatic Elastase. In Methods in Enzymology. Colowick et Kaplan Éd., V, 665-73. Acad. Press. New York.

NAGASE G., I964. Contribution to the physiology of digestion in Tilapia mossambica PETERs : Digestive enzymes and the effect of diets on their activity. $Z$. Vergl. Physiol., 49, 270-284.

Nagayama F., Saito Y., r969. Distribution of several hydrolytic enzymes in fish. EIFAC Tech. Paper, 9, I03-II6.

Nilsson A., FANGe R., rg69. Digestive proteases in the holocephalian fish, Chimaera monstruosa L. Comp. Biochem. Physiol., 31, r47-165.

Nilsson A., Fange R., r97o. Digestive proteases in the cyclostome Myxine glutinosa L. Comp. Biochem. Physiol., 32, 237-250.

Norris E. R., Elam D., I940. Preparation and properties of cristalline salmon pepsin. J. biol. Chem., 134, 443-454.

Norris E. R., Mathies J. C., I953. Preparation and properties and cristallization of tuna pepsin. $J$. biol. Chem., 204, 673-686.

Ooshiro Z., I97I. Studies on proteinase in pyloric coeca of Fishes. II. Some properties of proteinase purified from the pyloric coeca of mackerel. Bull. Jap. Soc. sci. Fish., 37, r45-148.

VEGas-Velez M., I972. La structure histologique du tube digestif des poissons téléostéens. Tethys, 4, I63-I74.

Vonk H. J., 1955. Comparative physiologye : nutrition, feeding and digestion. Ann. Rev. Physiol., 17, $483-498$.

ZENDZIAN E. N., BeRnaRd E. A., 1967. Distribution of pancreatic ribonuclease, chymotrypsin and trypsin in vertebrates. Arch. Biochem. Biophys., 122, 699-713. 